Article

\title{
New Stability Criteria for Discrete Linear Systems Based on Orthogonal Polynomials
}

\author{
Luis E. Garza ${ }^{1, *+\dagger}$ (D) Noé Martínez ${ }^{2,+}$ and Gerardo Romero ${ }^{2,+}$ (iD) \\ 1 Facultad de Ciencias, Universidad de Colima, Colima 28045, Mexico \\ 2 Unidad Académica Multidisciplinaria Reynosa Rodhe, Universidad Autónoma de Tamaulipas, \\ Reynosa 88779, Mexico; a2193728004@alumnos.uat.edu.mx (N.M.); gromero@docentes.uat.edu.mx (G.R.) \\ * Correspondence: luis_garza1@ucol.mx \\ + These authors contributed equally to this work.
}

Received: 15 July 2020; Accepted: 6 August 2020; Published: 8 August 2020

\begin{abstract}
A new criterion for Schur stability is derived by using basic results of the theory of orthogonal polynomials. In particular, we use the relation between orthogonal polynomials on the real line and on the unit circle known as the Szegó transformation. Some examples are presented.
\end{abstract}

Keywords: orthogonal polynomials on the unit circle; Schur polynomials; Hurwitz polynomials; Szeg̋̋ transformation

MSC: 42C05, 93C05

\section{Introduction}

\subsection{Stability of Linear Systems}

The stability problem of dynamical systems is of great interest because of its numerous applications, mainly in control systems. This property is very important because of both its relationship to the good performance of dynamical systems and the prevention of their physical damage. It is well known that for time-invariant linear dynamical systems, stability is determined by the roots of a characteristic equation that has a polynomial form. In the case of continuous systems, they are said to be stable if their roots have negative real part. This is known as Hurwitz stability. Now, for the discrete case, the system is stable if its roots are within a circle of radius equal to 1 , this is known as Schur stability. Polynomials that satisfy the first condition are called Hurwitz polynomials, while those that satisfy the last one are called Schur polynomials. Therefore, the problem of verifying the stability property in dynamical systems is transformed into verifying the Hurwitz or Schur properties of polynomials. This contribution will address the problem of verifying the Schur stability property. The most common strategy to verify the Schur stability property is using a bilinear transformation (Möbius transformation, see [1,2]) and then applying Hurwitz stability tools to solve the original problem. However, [3] shows that their application is restricted by a pathological case and so the authors propose to use a biquadratic transformation to avoid this problem. Many papers that address this problem have been published, for example [4] presents conditions to verify the Schur stability property for the particular cases of real $2 \times 2$ matrices and real $n \times n$ tridiagonal matrices for which the concepts Schur D-stable and vertex stable are introduced and verified. In [5] some results are presented to determine regions defined by the gains of PID controllers that guarantee the stability property of discrete systems, those results use the bilinear transformation to exploit results of continuous systems and the linear programming technique to delimit the regions. On the other hand, there is another method that is also used to verify the Schur stability property, known as the Jury test (see [6,7]). A simplified proof for this test is presented in [8]. 
Another topic that has also been studied consists of verifying the Schur stability property by considering uncertainty in the polynomial coefficients, which is known as robust Schur stability. Some of the first results related to this topic were the extension of Kharitonov-like results using, in some of them, the Möbius transformation in order to transform the problem into a robust Hurwitz stability problem, see $[9,10]$. Other criteria used to verify the robust Schur stabiliy of a family of polynomials are Schur-Cohn and the Vandermonde matrix, see [11-14]. Also, there are other papers that deal with the verification of robust Schur stability of interval matrices by using the spectral radius to determine the stability conditions, see $[15,16]$. Other results use multivariate polynomials to verify the robust Schur stability property. For example [17] establishes conditions to use the Möbius transformation in multivariate polynomials, while in [18] the authors first transform the problem into verifying the positivity of particularly defined bivariate functions and then they use semi-defined programming. The main disadvantage of the latter method is that it presents only sufficient conditions of robust Schur stability. The Schur stability property of polynomials depends directly on their coefficients, thus it is possible to define regions in the coefficient space for which the polynomial meets the condition of Schur stability. These regions are generally represented by semi-algebraic sets, which in turn, makes it possible to use criteria such as the Jury test. The paper [19] introduces the concept of polynomial superlevel sets which are used as a tool to construct approximations of the semi-algebraic sets that may be applied in control problems, in particular, the Hurwitz and Schur stability properties.

Finally, in this paper we present a new criterion to verify the Schur stability property. This is derived by using the relation between orthogonal polynomials on the real line and on the unit circle known as the Szego transformation. It is worth noting that this new approach to verify the Schur stability property can be generalized to define new semi-algebraic sets to be used in control theory. This approach is akin to the one presented in [19] but it has not been used before.

The structure of the manuscript is as follows. The remainder of this section contains some basic mathematical background on stable polynomials. Section 2 deals with basic results for orthogonal polynomials on the real line and on the unit circle that will be used in the sequel. The main contribution of our manuscript is contained in Section 3. There, we state a new criterion for Schur stability whose proof is based in orthogonality properties, and uses a well known mapping between the unit circle and the interval $[-2,2]$. Some illustrative examples are presented.

\subsection{Stable Polynomials}

A continuous linear system is stable if and only if its characteristic polynomial is a Hurwitz polynomial, i.e., the real part of all its zeros is strictly negative. As a consequence, Hurwitz polynomials are widely studied in the literature (see, for instance, $[20,21]$ ), and there are many criteria to determine if a given polynomial with real coefficients is Hurwitz without explicitly computing its zeros. Among many others, we have the Routh-Hurwitz criterion [20], the stability test [22], the continued fraction method [20], and the Hermite-Biehler theorem [23]. The latter determines the Hurwitz character of a polynomial $f(x)$ by verifying some properties of two polynomials associated with $f$. Indeed, if $h$ and $g$ are polynomials given by $f(x)=h\left(x^{2}\right)+x g\left(x^{2}\right)$, i.e., the even and odd parts of $f$, then the Hermite-Biehler theorem states that $f$ is a Hurwitz polynomials if and only if $h$ and $g$ have real, negative and interlaced zeros. Moreover, if we consider the series expansions

$$
\begin{aligned}
& \underline{g(x)}=\left\{\frac{s_{0}}{x}-\frac{s_{1}}{x^{2}}+\frac{s_{2}}{x^{3}}-\ldots+\frac{s_{2 n-2}}{x^{2 n-1}}-\frac{s_{2 n-1}}{x^{2 n}}+\ldots, \quad \text { if } \operatorname{deg}(f)\right. \text { is even, }
\end{aligned}
$$

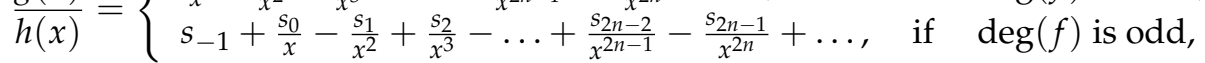


then the constants $\left\{s_{0}, s_{1}, \ldots, s_{2 n-1}\right\}$ and $\left\{s_{-1}, s_{0}, s_{1}, \ldots, s_{2 n-1}\right\}$ are known as Markov's parameters and it is known (see [20]) that $f$ is a Hurwitz polynomial if and only if the matrices

$$
H_{n-1}=\left(\begin{array}{cccc}
s_{0} & s_{1} & \ldots & s_{n-1} \\
s_{1} & s_{2} & \ldots & s_{n} \\
\vdots & \vdots & \vdots & \vdots \\
s_{n-1} & s_{n} & \ldots & s_{2 n-2}
\end{array}\right), \quad H_{n-1}^{(1)}=\left(\begin{array}{cccc}
s_{1} & s_{2} & \ldots & s_{n} \\
s_{2} & s_{3} & \ldots & s_{n+1} \\
\vdots & \vdots & \vdots & \vdots \\
s_{n} & s_{n+1} & \ldots & s_{2 n-1}
\end{array}\right)
$$

are both positive definite and $s_{-1}>0$ in the odd case.

On the other hand, a discrete linear system is stable if and only if its characteristic polynomial is a Schur polynomial, i.e., all of its zeros are located in the open unit disc $\mathbb{D}=\{z \in \mathbb{C}:|z|<1\}$. As we will see in a moment, the Hermite-Bielher theorem can be extended to this situation. More generally, an interlacing theorem can be stated for any stability region $A \subset \mathbb{C}$ such that the phase of any polynomial stable with respect to $A$ varies monotonically through the boundary $\partial A$ (see [22]).

In this section, we consider polynomials with real coefficients, unless otherwise stated. Without loss of generality, we will consider monic polynomials. Clearly, if

$$
S_{n}(z)=z^{n}+p_{n-1} z^{n-1}+\ldots+p_{1} z+p_{0}
$$

a necessary condition for $S_{n}(z)$ to be a Schur polynomial is that $\left|p_{0}\right|<1$. On the other hand, a necessary and sufficient condition for $S_{n}(z)$ to be a Schur polynomial is that the graph of $S_{n}\left(e^{j \theta}\right)$ circles $n$ times around the origin or, equivalently, the graph of $e^{j n \theta} S_{n}\left(e^{-j \theta}\right)$ does not circle around the origin when $\theta$ varies from 0 to $2 \pi$ (see [22]). Notice that this result is valid even for polynomials with complex coefficients. For the case of real coefficients, consider the polynomials

$$
\begin{gathered}
R(\theta)=\cos (n \theta)+p_{n-1} \cos ((n-1) \theta)+\ldots+\cos (\theta) p_{1}+p_{0} \\
I(\theta)=\sin (n \theta)+p_{n-1} \sin ((n-1) \theta)+\ldots+\sin (\theta) p_{1},
\end{gathered}
$$

known as the real and imaginary parts of $S_{n}(z)$. The last criteria can be used to show that $S_{n}(z)$ (with $\left|p_{0}\right|<1$ ) is a Schur polynomial if and only if (see [22])

(i) $\quad R(\theta)$ has exactly $n$ zeros in $[0, \pi]$,

(ii) $I(\theta)$ has exactly $n+1$ zeros in $[0, \pi]$,

(iii) The zeros of $R(\theta)$ and $I(\theta)$ interlace.

Another interlacing theorem is the following. Let $S_{n}(z)=S_{n}^{s}(z)+S_{n}^{a}(z)$, where

$$
S_{n}^{s}(z)=\frac{1}{2}\left[S_{n}(z)+z^{n} S_{n}\left(\frac{1}{z}\right)\right], \quad S_{n}^{a}(z)=\frac{1}{2}\left[S_{n}(z)-z^{n} S_{n}\left(\frac{1}{z}\right)\right] .
$$

Then, $S_{n}(z)$ is a Schur polynomial if and only if

(i) $S_{n}^{s}(z)$ and $S_{n}^{a}(z)$ are polynomials of degree $n$ with coefficients of the same sign.

(ii) $S_{n}^{s}(z)$ and $S_{n}^{a}(z)$ have simple and interlaced zeros on the unit circle $\mathbb{T}=\{z \in \mathbb{C}:|z|=1\}$.

Notice that, since the coefficients are real, the zeros of $S_{n}^{s}(z)$ and $S_{n}^{a}(z)$ appear in conjugate pairs. The polynomial $z^{n} S_{n}\left(\frac{1}{z}\right)$ is usually called the reciprocal (or reversed) polynomial of $S_{n}(z)$ and it is denoted by $S_{n}^{*}(z)$. We say that a polynomial $A(z)$ is symmetric if $A(z)=A^{*}(z)$ and anti-symmetric if $A(z)=-A^{*}(z)$. This explains the notation in (2) since $S_{n}^{s}(z)$ and $S_{n}^{a}(z)$ are clearly symmetric and anti-symmetric polynomials, respectively. The previous criterion can be generalized as follows.

Theorem 1 ([24]). Let $S_{n}(z)$ be a real Schur polynomial and $k \geq 0$. Then, the polynomials $\mathcal{A}$ and $\mathcal{B}$ defined by

$$
\begin{aligned}
& \mathcal{A}(z)=S_{n}(z)+z^{-k} S_{n}^{*}(z), \\
& \mathcal{B}(z)=S_{n}(z)-z^{-k} S_{n}^{*}(z),
\end{aligned}
$$


have interlaced zeros in $\mathbb{T}$ and $\mathcal{A}$ has a conjugate pair of zeros closer to $z=1$. Conversely, if $A$ and $B$ are any two real polynomials of the same degree, such that one is symmetric and the other is anti-symmetric, with interlaced zeros on $\mathbb{T}$, then $A(z)+B(z)$ is a Schur polynomial.

A different criterion to determine whether or not a given polynomial is a Schur polynomial uses a recursive algorithm, as follows. Notice that it is equivalent to the Jury criteria, and its proof is based in the so-called Boundary Crossing Theorem (see [6,7], and also [22]). It constitutes a discrete analogue of the Routh-Hurwitz stability criterion.

Observe that each application of step 5 results in a 1 degree reduction in the computed polynomial, with respect to the previous one. Thus, after $n-1$ cycles the resulting polynomial has degree 1 .

Finally, it is well known that the Möbius (also called bilinear) transformation $z=\frac{x+1}{x-1}$ maps the open unit disc into the open left half plane. Thus, it can be used to transform a polynomial $S_{n}(z)$ into a polynomial $f_{n}(x)$ by using

$$
(x-1)^{n} S_{n}\left(\frac{x+1}{x-1}\right)=f_{n}(x) .
$$

Then, if the leading coefficient of $f_{n}$ is not zero, the transformation preserves the degree and in this situation $S(z)$ is a Schur polynomials if and only if $f(x)$ is a Hurwitz polynomial [22]. As a consequence, it is possible to determine if a given polynomial is Schur by using Hurwitz criteria.

\section{Orthogonal Polynomials and the Szegő Transformation}

\subsection{Orthogonal Polynomials on the Real Line}

Let $\mu$ be a positive measure supported in some subset $E \subset \mathbb{R}$ with infinite points and such that

$$
\left|\int_{E} x^{k} d \mu(x)\right|<\infty, \quad k \geq 0 .
$$

If $\mu(x)$ is an absolutely continuous measure, then we can write $d \mu(x)=\omega(x) d x$, where $\omega(x)$ is an integrable, non-negative function such that $\int_{E} \omega(x) d x>0$. It is well known that there exists a sequence of real polynomials $\left\{p_{n}\right\}_{n \geq 0}$ such that

$$
\int_{E} p_{n}(x) p_{m}(x) d \mu(x)=\gamma_{n} \delta_{n, m}, \quad \gamma_{n}>0, \quad n, m \geq 0,
$$

where $\delta_{m, n}$ is the Kronecker's delta. $\left\{p_{n}\right\}_{n \geq 0}$ is called the sequence of polynomials orthogonal with respect to $\mu$ (or with respect to the weight function $\omega$ ) and it is unique up to constant multiplications (see [25]). If $\gamma_{n}=1$ for every $n$, then $\left\{p_{n}\right\}_{n \geq 0}$ is called an orthonormal sequence. We can also consider a monic sequence $\left\{P_{n}\right\}_{n \geq 0}$ by dividing each $p_{n}(x)$ by its leading coefficient. This is the normalization that will be used in the manuscript.

Orthogonal polynomials on the real line have many applications and many useful properties. In particular, it is well known that each $P_{n}(x)$ has real and simple zeros, which are located in the interior of the convex hull of $E$. Moreover, if we denote by $x_{n, k}$ the zeros of $P_{n}(x)$, then they satisfy the following interlacing property (see [26])

$$
x_{n, k}<x_{n-1, k}<x_{n, k+1}, \quad 1 \leq k \leq n-1 .
$$

Furthermore, for any polynomial $P_{n}$ of degree $n$ with real and simple zeros, it is possible to find a positive measure $\mu$ such that $P_{n}$ belongs to a sequence of polynomials orthogonal with respect to $\mu$, although $P_{n-1}, P_{n-2}, \ldots, P_{1}, P_{0}$ are not uniquely determined. However, if $P_{n}$ and $P_{n-1}$ are arbitrary polynomials with degree $n$ and $n-1$, respectively, whose zeros are real, simple and interlaced, then there exists a measure $\mu$ such that they are orthogonal with respect to $\mu$, and $P_{n}, P_{n-1}, P_{n-2}, \ldots, P_{0}$ are uniquely determined. This is known as the Geronimus-Wendroff Theorem (see [26,27]). 
On the other hand, the location of the zeros of orthogonal polynomials has also been studied when the orthogonality measure is perturbed. In the literature, the case when the measure is multiplied by a polynomial is called the Christoffel transformation. Let $\alpha(x)$ be a polynomial which is non-negative in $E$ of the form

$$
\alpha(x)=\prod_{k=1}^{m}\left(x-x_{k}\right) .
$$

If we have $x_{k} \neq x_{j}$ for $k \neq j$, then the (monic) polynomials $P_{n}^{[m]}(x)$ defined by

$$
A_{n, m} \alpha(x) P_{n}^{[m]}(x)=\left|\begin{array}{cccc}
P_{n}\left(x_{1}\right) & P_{n+1}\left(x_{1}\right) & \ldots & P_{n+m}\left(x_{1}\right) \\
P_{n}\left(x_{2}\right) & P_{n+1}\left(x_{2}\right) & \ldots & P_{n+m}\left(x_{2}\right) \\
\vdots & \vdots & \vdots & \vdots \\
P_{n}\left(x_{m}\right) & P_{n+1}\left(x_{m}\right) & \ldots & P_{n+m}\left(x_{m}\right) \\
P_{n}(x) & P_{n+1}(x) & \ldots & P_{n+m}(x)
\end{array}\right|
$$

with

$$
A_{n, m}=\left|\begin{array}{cccc}
P_{n}\left(x_{1}\right) & P_{n+1}\left(x_{1}\right) & \ldots & P_{n+m-1}\left(x_{1}\right) \\
P_{n}\left(x_{2}\right) & P_{n+1}\left(x_{2}\right) & \ldots & P_{n+m-1}\left(x_{2}\right) \\
\vdots & \vdots & \vdots & \vdots \\
P_{n}\left(x_{m}\right) & P_{n+1}\left(x_{m}\right) & \ldots & P_{n+m-1}\left(x_{m}\right)
\end{array}\right|
$$

are orthogonal with respect to the perturbed measure $\alpha(x) d \mu(x)$ in $E$, and $P_{n}^{[m]}(x)$ has degree $n$. In particular, if $\left\{x_{n, k}^{[1]}\right\}_{k=0}^{n}$ denote the zeros of the polynomial $P_{n}^{[1]}(x)$ orthogonal with respect to $(x-c) d \mu(x)$ on $E=[a, b]$, then the zeros of the polynomials $P_{n}^{[1]}(x), P_{n}(x)$ and $P_{n+1}(x)$ satisfy the following interlacing property (see [26], where the polynomials $\left\{P_{n}^{[1]}\right\}_{n \geq 0}$ are called kernel polynomials $[28,29])$ :

- If $c \leq a$, then

$$
x_{n+1,1}<x_{n, 1}<x_{n, 1}^{[1]}<x_{n+1,2}<\cdots<x_{n, n}<x_{n, n}^{[1]}<x_{n+1, n+1}
$$

- If $c \geq b$, then

$$
x_{n+1,1}<x_{n, 1}^{[1]}<x_{n, 1}<\cdots<x_{n+1, n}<x_{n, n}^{[1]}<x_{n, n}<x_{n+1, n+1} .
$$

Finally, we point out that there is a close relation between Hurwitz polynomials and orthogonal polynomials on the real line. Indeed, any Hurwitz polynomial can be expressed in terms of an orthogonal polynomial and its associated polynomial (see [26]). Conversely, it is possible to construct a Hurwitz polynomial by using orthogonal polynomials. For more details, we refer the reader to [20,30-33] and, more recently, [34]. In the latter, the authors construct sequences of Hurwitz polynomials from a sequence of orthogonal polynomials, and show several algebraic properties of the constructed family. Also, classical orthogonal polynomials are used in [35] to construct families of Hurwitz polynomials that are robustly stable.

\subsection{Orthogonal Polynomials on the Unit Circle}

Let $\sigma$ be a positive, non-trivial measure supported on the unit circle $\mathbb{T}$. Generally, $\sigma$ is assumed to be a probability measure, i.e., $\int_{\mathbb{T}} d \sigma(z)=1$. Then, there exists a sequence of complex polynomials $\left\{\phi_{n}\right\}_{n \geq 0}$ with $\operatorname{deg}\left(\phi_{n}\right)=n$ such that

$$
\int_{\mathbb{T}} \phi_{n}(z) \overline{\phi_{m}(z)} d \sigma(z)=k_{n} \delta_{n, m}, \quad \forall n, m \geqslant 0,
$$

where $k_{n}>0$ for every $n \geq 0$. We will assume that they are monic. $\left\{\phi_{n}\right\}_{n \geq 0}$ is called the monic sequence of polynomials orthogonal with respect to $\sigma$, and satisfies the following recurrence relations $[25,27]$ 


$$
\phi_{n+1}(z)=z \phi_{n}(z)+\phi_{n+1}(0) \phi_{n}^{*}(z)
$$

(ii) Backward recurrence:

$$
\phi_{n+1}(z)=\left(1-\left|\phi_{n+1}(0)\right|^{2}\right) z \phi_{n}(z)+\phi_{n+1}(0) \phi_{n+1}^{*}(z),
$$

where $\phi_{n}^{*}(z)=z^{n} \overline{\phi_{n}}\left(z^{-1}\right)$.

Notice that $\phi_{n}^{*}(z)$ is the reciprocal polynomial as defined in the previous section, except that here the coefficients are in general complex and the conjugate has to be taken. The complex numbers $\left\{\phi_{n}(0)\right\}_{n \geq 0}$ are called Verblunsky (Schur, reflection) coefficients, and satisfy $\left|\phi_{n}(0)\right|<1$ for every $n \geq 1$.

It is well known that every positive, nontrivial measure $\sigma$ in $\mathbb{T}$ determines a unique sequence of Verblunsky coefficients $\left\{\phi_{n}(0)\right\}_{n \geq 1}$. Conversely, given an arbitrary sequence $\left\{a_{n}\right\}_{n \geq 1}$ of complex numbers satisfying $\left|a_{n}\right|<1$ for $n \geq 1$, there exists a unique measure $\sigma$ supported on $\mathbb{T}$ such that its corresponding monic orthogonal sequence $\left\{\phi_{n}(z)\right\}_{n \geq 1}$ satisfies $\phi_{n}(0)=a_{n}$ for $n \geq 1$. In other words, any complex sequence $\left\{a_{n}\right\}_{n>1}$ in $\mathbb{D}$ is the sequence of Verblunsky coefficients for some measure $\sigma$. This is known as Verblunsky's theorem (see [27]).

Moreover, the zeros of each $\phi_{n}(z)$ are located in $\mathbb{D}$. Conversely, given any complex polynomial $\phi_{n}(z)$ with zeros in $\mathbb{D}$, it is possible to find a measure $\sigma$ supported in $\mathbb{T}$ such that $\phi_{n}(z)$ is orthogonal with respect to $\sigma$. Furthermore, the previous polynomials in the sequence, $\phi_{n-1}, \ldots, \phi_{0}$, are completely determined. This is a consequence of the backward recurrence relation. Indeed, this is the unit circle analogue of the Wendroff-Geronimus theorem discussed above (see [27]). Notice that this implies that any Schur polynomial is an orthogonal polynomial, and vice versa. This is an important difference with respect to the relation between Hurwitz and orthogonal polynomials on the real line.

\subsection{The Szegő Transformation}

The Szegő transformation establishes a relation between orthogonal polynomials on the real line and on the unit circle by defining a correspondence between measures supported on the interval $[-2,2]$ and measures supported on $\mathbb{T}$ (see $[25,27,36])$.

The mappings $z=e^{i \theta} \mapsto 2 \cos (\theta)$, with $\theta \in[0,2 \pi)$ and $x \mapsto \arccos (x / 2)$ define a two-one correspondence between $\mathbb{T}$ and $[-2,2]$, that can be used to define a mapping between probability measures supported in $\mathbb{T}$ and probability measures supported in $[-2,2]$. If we restrict this mapping to measures in $\mathbb{T}$ that are even, i.e., $d \sigma(\theta)=d \sigma(-\theta)$, then the correspondence is one to one. Such measures are called symmetric. This mapping is commonly referred to as the Szegó transformation and it is denoted by $S z$. More precisely, we say $d \mu=S z(d \sigma)$ if and only if $d \sigma(\theta)=d \sigma(-\theta)$ and

$$
\int_{0}^{2 \pi} f(\theta) d \sigma(\theta)=\int_{-2}^{2} f(\operatorname{arcos}(x / 2)) d \mu(x)
$$

for any function $f$ such that $f(\theta)=f(-\theta)$. Notice that because of the symmetry we have [36]

$$
d \sigma \text { is even } \Leftrightarrow \overline{\phi_{n}(z)}=\phi_{n}(\bar{z}) \Leftrightarrow \phi_{n}(0) \in \mathbb{R}
$$

for all $n \geq 1$. That is, the sequence $\left\{\phi_{n}\right\}_{n \geq 0}$ orthogonal with respect to $\sigma$ has real coefficients and, in particular, real Verblunsky coefficients. The next theorem establishes a relation between the sequences of orthogonal polynomials associated with $\mu$ and $\sigma$, when they are related through the Szegó transformation. The proof can be found in $[25,36]$. 
Theorem 2. Let $d \mu=S z(d \sigma)$, and denote by $\left\{P_{n}\right\}_{n \geq 0}$ and $\left\{\phi_{n}\right\}_{n \geq 0}$ the monic orthogonal sequences associated with $\mu$ and $\sigma$, respectively. Then,

$$
\begin{aligned}
P_{n}\left(z+\frac{1}{z}\right) & =\left[1+\phi_{2 n}(0)\right]^{-1} z^{-n}\left[\phi_{2 n}(z)+\phi_{2 n}^{*}(z)\right], \quad n \geq 0 \\
\left\|P_{n}\right\|_{L^{2}(d \mu)}^{2}=2\left[1+\phi_{2 n}(0)\right]^{-1}\left\|\phi_{2 n}\right\|_{L^{2}(d \sigma)^{\prime}}^{2} \quad n \geq 0 & \\
P_{n}\left(z+\frac{1}{z}\right) & =z^{-n}\left[z \phi_{2 n-1}(z)+\phi_{2 n-1}^{*}(z)\right], \quad n \geq 1 \\
\left\|P_{n}\right\|_{L^{2}(d \mu)}^{2} & =2\left[1-\phi_{2 n}(0)\right]\left\|\phi_{2 n-1}\right\|_{L^{2}(d \sigma)^{\prime}}^{2} \quad n \geq 1
\end{aligned}
$$

where $L^{2}(d \mu)$ is the space of measurable functions such that $\int_{[-2,2]}|f(x)|^{2} d \mu(x)<\infty$, and $L^{2}(d \sigma)$ is defined in a similar way.

There is a second family related to $\left\{\phi_{n}\right\}_{n \geq 0}$, orthogonal with respect to the measure

$$
d \mu_{1}(x)=\frac{1}{4}\left(4-x^{2}\right) d \mu(x) .
$$

If we denote by $\left\{Q_{n}(x)\right\}_{n \geq 0}$ the corresponding monic orthogonal polynomials, we have for $n \geq 1$

$$
\begin{aligned}
Q_{n-1}\left(z+\frac{1}{z}\right) & =\left(1-\phi_{2 n}(0)\right)^{-1} z^{-n} \frac{\phi_{2 n}(z)-\phi_{2 n}^{*}(z)}{z-z^{-1}} \\
& =z^{-n} \frac{z \phi_{2 n-1}(z)-\phi_{2 n-1}^{*}(z)}{z-z^{-1}}
\end{aligned}
$$

and, from Equations (15), (17) and (19) we have

$$
\begin{aligned}
\phi_{2 n}(z) & =\frac{z^{n}}{2}\left[\left(1+\phi_{2 n}(0)\right) P_{n}\left(z+\frac{1}{z}\right)+\left(1-\phi_{2 n}(0)\right)\left(z-z^{-1}\right) Q_{n-1}\left(z+\frac{1}{z}\right)\right], \\
\phi_{2 n-1}(z) & =\frac{z^{n-1}}{2}\left[P_{n}\left(z+\frac{1}{z}\right)+\left(z-z^{-1}\right) Q_{n-1}\left(z+\frac{1}{z}\right)\right] .
\end{aligned}
$$

\section{Stability Criteria via Orthogonality}

In this section, we use the basic results of the previous section to study stability criteria for Schur polynomials. First, notice that the expression in step 5 of Algorithm 1 is equivalent to the Szegó backward relation Equation (14). Thus, by applying Algorithm 1 to a polynomial of degree $n$ with complex coefficients we are in fact computing a sequence $\left\{S_{k}\right\}_{k=0}^{n}$ of polynomials that satisfy the Szegő recursion. The criterion asserts that the initial polynomial is Schur if and only if we have $\left|S_{k}(0)\right|<1$ for $k=1, \ldots, n$. Thus, Verblunsky's theorem and the analogue of the Geronimus-Wendroff theorem for the unit circle directly lead to another proof for the validity of the algorithm. This was already discussed in [27]. 


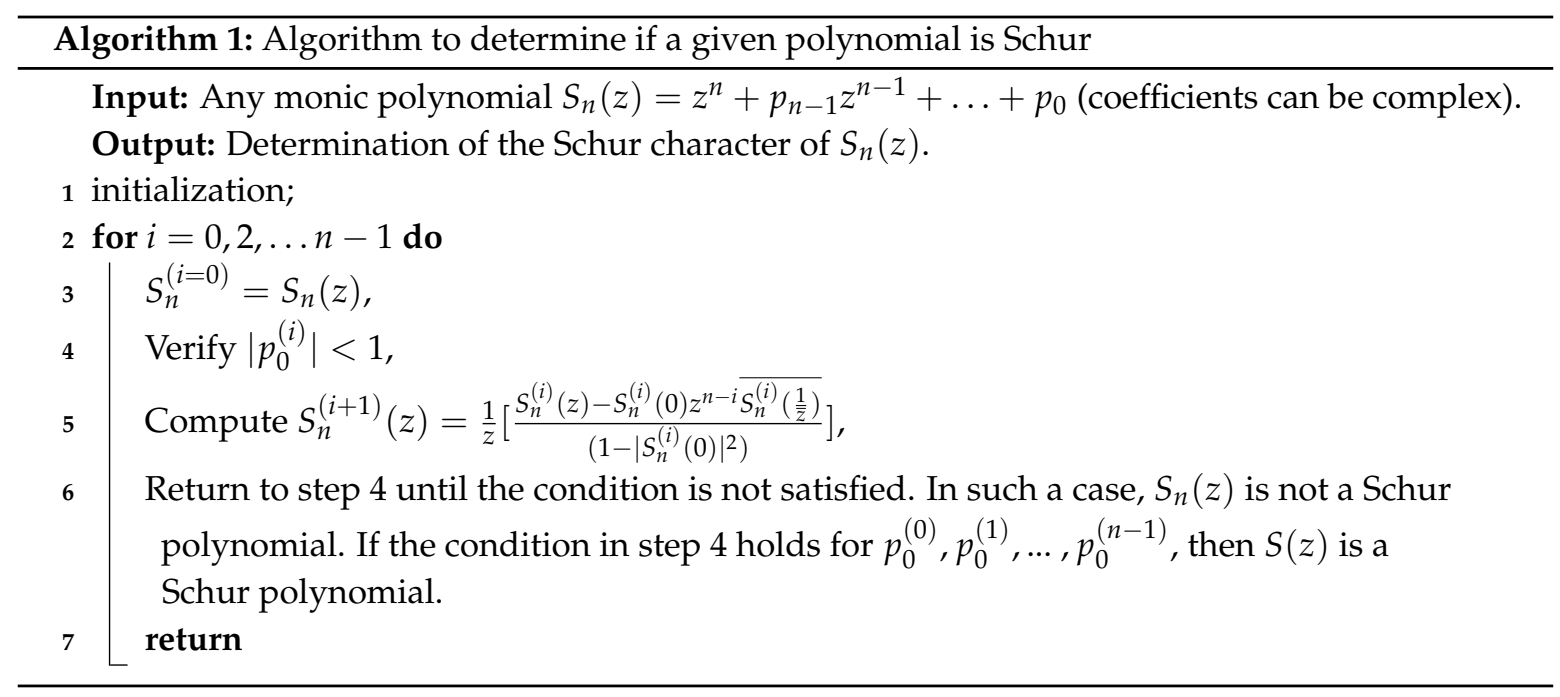

Before stating our main result, we need the following lemma.

Lemma 1. Let $\left\{P_{n}(x)\right\}_{n \geq 0}$ be the sequence of monic orthogonal polynomials with respect to a measure $d \mu$ supported on the interval $E=[-c, c]$, for some $c>0$, and denote by $\left\{P_{n}^{[2]}(x)\right\}_{n \geq 0}$ the monic orthogonal sequence with respect to $d \mu^{[2]}=\left(x^{2}-c^{2}\right) d \mu$. Then, if we denote by $\left\{x_{n, k}^{[2]}\right\}_{k=1}^{n}$ the zeros of $P_{n}^{[2]}(x)$ and by $\left\{x_{n+1, k}\right\}_{k=1}^{n+1}$ the zeros of $P_{n+1}(x)$, we have the interlacing property

$$
x_{n+1, k}<x_{n, k}^{[2]}<x_{n+1, k+1}, \quad 1 \leq k \leq n .
$$

Proof. Denote by $\left\{x_{n, k}^{[1]}\right\}_{k=0}^{n}$ the zeros of $P_{n}^{[1]}(x)$, orthogonal with respect to the measure $d \mu^{[1]}=(x-c) d \mu$. Then, they satisfy the interlacing property Equation (11). Since $\left\{P_{n}^{[2]}(x)\right\}_{n \geq 0}$ is orthogonal with respect to $d \mu^{[2]}=(x+c) d \mu^{[1]}$, then from Equation (10) we see that the zeros $\left\{x_{n, k}^{[2]}\right\}_{k=0}^{n}$ of $P_{n}^{[2]}(x)$ satisfy

$$
x_{n+1,1}^{[1]}<x_{n, 1}^{[1]}<x_{n, 1}^{[2]}<x_{n+1,2}^{[1]}<\ldots<x_{n, n}^{[1]}<x_{n, n}^{[2]}<x_{n+1, n+1}^{[1]} .
$$

As a consequence, we have

$$
x_{n+1,1}<x_{n, 1}^{[2]}<x_{n+1,2}<\ldots<x_{n+1, n}<x_{n, n}^{[2]}<x_{n+1, n+1} .
$$

As mentioned before, a useful tool to determine the Schur character of a polynomial is the Möbius transformation from $\mathbb{D}$ to the left half-plane of the complex plane. Then, the problem of determining the Schur stability becomes a problem of determining Hurwitz stability. Our next result establishes a novel criterion to determine Schur stability by using the Szegó transformation defined in the previous section.

Theorem 3. Let $S_{m}(z)$ be a m-th degree monic polynomial with real coefficients satisfying $\left|S_{m}(0)\right|<1$. If $m=2 n$, define

$$
\begin{aligned}
P_{n}\left(z+\frac{1}{z}\right) & =\left[1+S_{2 n}(0)\right]^{-1} z^{-n}\left[S_{2 n}(z)+S_{2 n}^{*}(z)\right], \\
Q_{n-1}\left(z+\frac{1}{z}\right) & =\left[\left(1-S_{2 n}(0)\right)\left(z-z^{-1}\right)\right]^{-1} z^{-n}\left[S_{2 n}(z)-S_{2 n}^{*}(z)\right] .
\end{aligned}
$$




$$
\text { If } m=2 n-1, \text { define }
$$

$$
\begin{aligned}
P_{n}\left(z+\frac{1}{z}\right) & =z^{-n}\left[z S_{2 n-1}(z)+S_{2 n-1}^{*}(z)\right] \\
Q_{n-1}\left(z+\frac{1}{z}\right) & =\left(z-z^{-1}\right)^{-1} z^{-n}\left[z S_{2 n-1}(z)-S_{2 n-1}^{*}(z)\right] .
\end{aligned}
$$

Then, $S_{m}(z)$ is a Schur polynomial if and only if $P_{n}(x)$ and $Q_{n-1}(x)$ are real polynomials with real, simple, and interlaced roots in $(-2,2)$.

Proof. Notice that Equations (21)-(24) are Equations (15), (17) and (19). If $S_{m}(z)$ is a Schur polynomial with real coefficients, then it is orthogonal with respect to some even function $\sigma$ supported on $\mathbb{T}$. Then, by applying the Szegó transformation, we obtain a measure $\mu$ supported on $[-2,2]$, with an associated monic orthogonal sequence $\left\{P_{n}\right\}_{n \geq 0}$. Notice that the zeros of each $P_{n}$ are real, simple, and located in $(-2,2)$. Moreover, the sequence $\left\{Q_{n}\right\}_{n \geq 0}$ is orthogonal with respect to $\frac{1}{4}\left(4-x^{2}\right) d \mu$, and their zeros are also simple and lie in $(-2,2)$. By the previous lemma, $P_{n}(x)$ and $Q_{n-1}(x)$ have interlaced roots.

Conversely, assume that $P_{n}(x)$ and $Q_{n-1}(x)$ are real polynomials with real, simple and interlaced roots in $(-2,2)$. Then, $P_{n}\left(z+\frac{1}{z}\right)$ and $\left(z-z^{-1}\right) Q_{n-1}\left(z+\frac{1}{z}\right)$ clearly have simple and interlacing zeros in $\mathbb{T}$. If $m=2 n-1$ (resp. $m=2 n$ ), then it follows from Equations (17) and (19) (resp. from Equations (15) and (19)) that $\left[z S_{m}(z)+S_{m}^{*}(z)\right]$ and $\left[z S_{m}(z)-S_{m}^{*}(z)\right]$ (resp. $\left[S_{m}(z)+S_{m}^{*}(z)\right]$ and $\left.\left[S_{m}(z)-S_{m}^{*}(z)\right]\right)$ have interlaced zeros in $\mathbb{T}$. Notice that $\left[z S_{m}(z)+S_{m}^{*}(z)\right]$ is a symmetric polynomial and $\left[z S_{m}(z)-S_{m}^{*}(z)\right]$ is an anti-symmetric polynomial (resp. $\left[S_{m}(z)+S_{m}^{*}(z)\right]$ is symmetric and $\left[S_{m}(z)-S_{m}^{*}(z)\right]$ is anti-symmetric) and both polynomials have degree $m+1$ (resp. $m$ ). Since $z S(z)=\frac{1}{2}\left[z S_{m}(z)+S_{m}^{*}(z)+z S_{m}(z)-S_{m}^{*}(z)\right]\left(\operatorname{resp} . S(z)=\frac{1}{2}\left[S_{m}(z)+S_{m}^{*}(z)+S_{m}(z)-S_{m}^{*}(z)\right]\right)$ it follows from Theorem 1 that $S_{m}(z)$ is a Schur polynomial.

The following result follows at once. It establishes a necessary condition for the Schur character of a real polynomial.

Corollary 1. Let $S_{m}(z)$ be a $m$-th degree monic Schur polynomial with real coefficients. Define $P_{n}(x)$ as in the previous theorem. Then, $P_{n}(x+2)$ is a Hurwitz polynomial with zeros in $(-4,0)$.

In other words, given any real polynomial $S_{m}(z)$ of degree $m$, we can compute $P_{n}(x)$ as in the previous theorem and then check $P_{n}(x+2)$ for Hurwitz stability. If it is not Hurwitz, then $S_{m}(z)$ is not Schur. As another straightforward consequence, we can state the following Wendroff-Geronimus type theorem.

Corollary 2. Let $P_{n}(x)$ and $Q_{n-1}(x)$ be two monic, real polynomials with degree $n$ and $n-1$, respectively. If $P_{n}$ and $Q_{n-1}$ have real, simple and interlaced zeros in $(-2,2)$, then there exists a positive measure $\mu$ supported in $[-2,2]$ such that $P_{n}$ is orthogonal with respect to $\mu$ and $Q_{n-1}$ is orthogonal with respect to the Christoffel transformation $\left(x^{2}-4\right) d \mu$. Moreover, the orthogonal sequences $P_{n-1}, \ldots, P_{0}$ and $Q_{n-2}, \ldots, Q_{0}$ are uniquely determined.

Proof. If $P_{n}(x)$ and $Q_{n-1}(x)$ have simple and interlaced roots in $(-2,2)$, then the polynomial $\phi_{2 n-1}(z)$ obtained from Equation (20) is a Schur (and thus orthogonal) monic polynomial with real coefficients, from Theorem 3. By using Equations (23) and (24) for $\phi_{2 n-1}(z)$ we obtain again $P_{n}(x)$ and $Q_{n-1}(x)$, and therefore $P_{n}(x)$ is orthogonal with respect to some $\mu$ supported on $[-2,2]$ and $Q_{n-1}(x)$ is orthogonal with respect to $\left(x^{2}-4\right) d \mu$. The polynomials $P_{n-1}, \ldots, P_{0}$ and $Q_{n-2}, \ldots, Q_{0}$ are uniquely determined by the polynomials $\phi_{2 n-3}, \phi_{2 n-5}, \ldots, \phi_{1}$ obtained by applying the backward recurrence relation to $\phi_{2 n-1}(z)$. 
The following lemma shows how to compute the coefficients of the polynomials $P_{n}$ and $Q_{n-1}$ in Theorem 3. Notice that instead of computing the coefficients of $Q_{n-1}(x)$, we will find the coefficients of the polynomial $\left(x^{2}-4\right) Q_{n-1}(x)$, which has two additional zeros at $x= \pm 2$.

Lemma 2. Let $S_{m}(z)=z^{m}+p_{m-1} z^{m-1}+\ldots+p_{1} z+p_{0}$ and $P_{n}(x)=x^{n}+\gamma_{n-1} x^{n-1}+\ldots+\gamma_{1} x+\gamma_{0}$ be the polynomials in Theorem 3 . Then, the coefficients of $P_{n}(x)$ are determined by the linear system

$$
\left(\begin{array}{cccccccc}
\left(\begin{array}{l}
0 \\
0
\end{array}\right) & 0 & \left(\begin{array}{l}
2 \\
1
\end{array}\right) & 0 & \left(\begin{array}{l}
4 \\
2
\end{array}\right) & \ldots & \ldots & \\
0 & \left(\begin{array}{l}
1 \\
0
\end{array}\right) & 0 & \left(\begin{array}{l}
3 \\
1
\end{array}\right) & 0 & \ldots & \ldots & \\
0 & 0 & \left(\begin{array}{l}
2 \\
0
\end{array}\right) & 0 & \left(\begin{array}{l}
4 \\
1
\end{array}\right) & \ldots & \ldots & \\
\vdots & \vdots & \vdots & \ddots & \vdots & \vdots & \vdots & \vdots \\
0 & 0 & 0 & 0 & \ddots & 0 & \left(\begin{array}{c}
n-1 \\
1
\end{array}\right) & 0 \\
0 & 0 & 0 & 0 & \cdots & \left(\begin{array}{c}
n-2 \\
1
\end{array}\right) & 0 & \left(\begin{array}{c}
n \\
1
\end{array}\right) \\
0 & 0 & 0 & 0 & \cdots & 0 & \left(\begin{array}{c}
n-1 \\
0
\end{array}\right) & 0 \\
0 & 0 & 0 & 0 & \ldots & 0 & 0 & \left(\begin{array}{c}
n \\
0
\end{array}\right)
\end{array}\right)\left(\begin{array}{c}
\gamma_{0} \\
\gamma_{1} \\
\gamma_{2} \\
\vdots \\
\gamma_{n}
\end{array}\right)=\left(\begin{array}{c}
c_{0} \\
c_{1} \\
c_{2} \\
\vdots \\
c_{n}
\end{array}\right)
$$

where $c_{i}=\frac{1}{1+p_{0}}\left(p_{n+i}+p_{n-i}\right)$ for $0 \leq i \leq n$ if $m=2 n$, and $c_{i}=p_{n+i-1}+p_{n-i-1}$ for $0 \leq i \leq n$ if $m=2 n-1$, and defining $\gamma_{n}=p_{m}=1$ and $p_{-1}=0$. On the other hand, the coefficients of $\left(x^{2}-4\right) Q_{n-1}(x)=x^{n+1}+\eta_{n} x^{n}+\ldots+\eta_{1} x+\eta_{0}$ are given by the linear system

$$
\left(\begin{array}{cccccccc}
\left(\begin{array}{l}
0 \\
0
\end{array}\right) & 0 & \left(\begin{array}{l}
2 \\
1
\end{array}\right) & 0 & \left(\begin{array}{l}
4 \\
2
\end{array}\right) & \ldots & \ldots & \\
0 & \left(\begin{array}{l}
1 \\
0
\end{array}\right) & 0 & \left(\begin{array}{l}
3 \\
1
\end{array}\right) & 0 & \ldots & \ldots & \\
0 & 0 & \left(\begin{array}{l}
2 \\
0
\end{array}\right) & 0 & \left(\begin{array}{l}
4 \\
1
\end{array}\right) & \ldots & \ldots & \\
\vdots & \vdots & \vdots & \ddots & \vdots & \vdots & \vdots & \vdots \\
0 & 0 & 0 & 0 & \ddots & 0 & \left(\begin{array}{c}
n \\
1
\end{array}\right) & 0 \\
0 & 0 & 0 & 0 & \cdots & \left(\begin{array}{c}
n-1 \\
1
\end{array}\right) & 0 & \left(\begin{array}{c}
n+1 \\
1
\end{array}\right) \\
0 & 0 & 0 & 0 & \cdots & 0 & \left(\begin{array}{c}
n \\
0
\end{array}\right) & 0 \\
0 & 0 & 0 & 0 & \cdots & 0 & 0 & \left(\begin{array}{c}
n+1 \\
0
\end{array}\right)
\end{array}\right)\left(\begin{array}{c}
\eta_{0} \\
\eta_{1} \\
\eta_{2} \\
\vdots \\
\eta_{n+1}
\end{array}\right)=\left(\begin{array}{c}
-2 l_{1} \\
-l_{2} \\
-l_{3}+l_{1} \\
-l_{4}+l_{2} \\
\vdots \\
-l_{n-1}+l_{n-3} \\
-l_{n}+l_{n-2} \\
l_{n-1} \\
l_{n}
\end{array}\right)
$$

where $l_{i}=\frac{1}{1-p_{0}}\left(p_{n+i}-p_{n-i}\right)$ for $1 \leq i \leq n$ if $m=2 n$, and $l_{i}=p_{n+i-1}-p_{n-i-1}$ for $1 \leq i \leq n$ if $m=2 n-1$, with $\eta_{n+1}=1$.

Proof. Assume $S_{m}(z)$ has even degree $m=2 n$. Then, $P_{n}(x)$ has degree $n$ and we have

$$
P_{n}(z+1 / z)=\sum_{k=0}^{n} \gamma_{k}(z+1 / z)^{k}=\sum_{k=0}^{n} \gamma_{k} \sum_{j=0}^{k}\left(\begin{array}{l}
k \\
j
\end{array}\right) z^{k-2 j} .
$$

On the other hand, from Equation (21) we get

$$
\begin{aligned}
P_{n}(z+1 / z)= & \frac{1}{p_{0}+1}\left[\left(p_{0}+p_{2 n}\right)\left(z^{n}+z^{-n}\right)+\left(p_{1}+p_{2 n-1}\right)\left(z^{n-1}+z^{-(n-1)}\right)\right. \\
& \left.+\left(p_{2}+p_{2 n-2}\right)\left(z^{n-2}+z^{-(n-2)}\right)+\cdots+2 p_{n}\right] .
\end{aligned}
$$


Thus, by comparing the coefficients of the positive powers in Equations (27) and (28) we easily get Equation (25). Now, applying the change of variable $x=z+1 / z$ in $\left(x^{2}-4\right) Q_{n-1}(x)$, we get $\left(z-z^{-1}\right)^{2} Q_{n-1}(z+1 / z)$. Then, from Equation (22) we obtain

$$
\begin{aligned}
\left(z-z^{-1}\right)^{2} Q_{n-1}(z+1 / z)= & \frac{1}{1-p_{0}}\left[\left(1-p_{0}\right)\left(z^{n+1}+z^{-(n+1)}\right)+\left(p_{2 n-1}-p_{1}\right)\left(z^{n}+z^{-n}\right)\right. \\
& +\left(p_{2 n-2}-p_{2}-\left(1-p_{0}\right)\right)\left(z^{n-1}+z^{-(n-1)}\right) \\
& +\left(p_{2 n-3}-p_{3}-\left(p_{2 n-1}-p_{1}\right)\right)\left(z^{n-2}+z^{-(n-2)}\right) \\
& +\cdots+\left(p_{n+1}-p_{n-1}-\left(p_{n+3}-p_{n-3}\right)\right)\left(z^{2}+z^{-2}\right) \\
& \left.+\left(p_{n-2}-p_{n+2}\right)\left(z+z^{-1}\right)+2\left(p_{n-1}-p_{n+1}\right)\right] .
\end{aligned}
$$

Thus, comparing coefficients in Equations (27) and (29) we obtain Equation (26). Notice that the matrix in the linear system has size $(n+2) \times(n+2)$ since the polynomial $\left(x^{2}-4\right) Q_{n-1}(x)$ has degree $n+1$. The odd case $m=2 n-1$ follows in a similar way.

Finally, we illustrate the criterion in Theorem 3 with the following examples. Notice that the matrices of the linear systems in the previous lemma are lower triangular and therefore their solutions can be computed efficiently.

Example 1. Consider the polynomial $S_{4}(z)=z^{4}-z^{3}+\frac{3}{4} z^{2}+z+\frac{1}{2}$. The corresponding linear system is

$$
\left(\begin{array}{ccc}
\left(\begin{array}{c}
0 \\
0
\end{array}\right) & 0 & \left(\begin{array}{l}
2 \\
1
\end{array}\right) \\
0 & \left(\begin{array}{l}
1 \\
0
\end{array}\right) & 0 \\
0 & 0 & \left(\begin{array}{l}
2 \\
0
\end{array}\right)
\end{array}\right)\left(\begin{array}{l}
\gamma_{0} \\
\gamma_{1} \\
\gamma_{2}
\end{array}\right)=\left(\begin{array}{l}
1 \\
0 \\
1
\end{array}\right),
$$

and has solution $(-1,0,1)^{t}$. On the other hand, the linear system

$$
\left(\begin{array}{cccc}
\left(\begin{array}{l}
0 \\
0
\end{array}\right) & 0 & \left(\begin{array}{l}
2 \\
1
\end{array}\right) & 0 \\
0 & \left(\begin{array}{c}
1 \\
0
\end{array}\right) & 0 & \left(\begin{array}{l}
3 \\
1
\end{array}\right) \\
0 & 0 & \left(\begin{array}{c}
2 \\
0
\end{array}\right) & 0 \\
0 & 0 & 0 & \left(\begin{array}{l}
3 \\
0
\end{array}\right)
\end{array}\right)\left(\begin{array}{l}
\eta_{0} \\
\eta_{1} \\
\eta_{2} \\
\eta_{3}
\end{array}\right)=\left(\begin{array}{c}
8 \\
-1 \\
-4 \\
1
\end{array}\right),
$$

has solution $(16,-4,-4,1)^{t}$. As a consequence, the polynomials $P_{2}(x)$ and $Q_{1}(x)$ of Theorem 3 are $P_{2}(x)=x^{2}-1$ and $\left(x^{2}-4\right) Q_{1}(x)=x^{3}-4 x^{2}-4 x+16$. Notice that $P_{2}(x)$ has zeros at $x= \pm 1$, and $\left(x^{2}-4\right) Q_{1}(x)$ has zeros at 2,-2 and 4. Thus, $S_{4}(z)$ is not a Schur polynomial. Indeed, $S(z)$ has zeros $z_{1} \approx-0.391713-0.335138 i, z_{2} \approx-0.391713+0.335138 i$, $z_{3} \approx 0.891713-1.04224 i, z_{4} \approx 0.891713+1.04224 i$.

Notice that the previous example shows that $P_{n}(x)$ having zeros in $(-2,2)$ is not sufficient to guarantee that $S_{2 n}(z)$ is a Schur polynomial.

Example 2. Consider $S_{11}(z)=z^{11}-\frac{9}{4} z^{10}+\frac{157}{32} z^{9}-\frac{55}{8} z^{8}+\frac{4637}{512} z^{7}-\frac{9485}{1024} z^{6}+\frac{8909}{1024} z^{5}-\frac{6717}{1024} z^{4}+\frac{2261}{512} z^{3}-$ $\frac{37}{16} z^{2}+\frac{31}{32} z-\frac{1}{4}$. Then, the linear system

$$
\left(\begin{array}{ccccccc}
\left(\begin{array}{l}
0 \\
0
\end{array}\right) & 0 & \left(\begin{array}{l}
2 \\
1
\end{array}\right) & 0 & \left(\begin{array}{l}
4 \\
2
\end{array}\right) & 0 & \left(\begin{array}{l}
6 \\
3
\end{array}\right) \\
0 & \left(\begin{array}{l}
1 \\
0
\end{array}\right) & 0 & \left(\begin{array}{l}
3 \\
1
\end{array}\right) & 0 & \left(\begin{array}{l}
5 \\
2
\end{array}\right) & 0 \\
0 & 0 & \left(\begin{array}{l}
2 \\
0
\end{array}\right) & 0 & \left(\begin{array}{l}
4 \\
1
\end{array}\right) & 0 & \left(\begin{array}{l}
6 \\
2
\end{array}\right) \\
0 & 0 & 0 & \left(\begin{array}{l}
3 \\
0
\end{array}\right) & 0 & \left(\begin{array}{c}
5 \\
1
\end{array}\right) & 0 \\
0 & 0 & 0 & 0 & \left(\begin{array}{l}
4 \\
0
\end{array}\right) & 0 & \left(\begin{array}{l}
6 \\
1
\end{array}\right) \\
0 & 0 & 0 & 0 & 0 & \left(\begin{array}{l}
5 \\
0
\end{array}\right) & 0 \\
0 & 0 & 0 & 0 & 0 & 0 & \left(\begin{array}{l}
6 \\
0
\end{array}\right)
\end{array}\right)\left(\begin{array}{l}
\gamma_{0} \\
\gamma_{1} \\
\gamma_{2} \\
\gamma_{3} \\
\gamma_{4} \\
\gamma_{5} \\
\gamma_{6}
\end{array}\right)=\left(\begin{array}{c}
2 \frac{8909}{1024} \\
-\frac{9485}{1024}-\frac{6717}{1024} \\
\frac{4637}{512}+\frac{2261}{512} \\
-\frac{55}{8}-\frac{37}{16} \\
\frac{157}{32}+\frac{31}{32} \\
-\frac{9}{4}-\frac{1}{4} \\
1
\end{array}\right)
$$


has solution $\left(\frac{105}{512},-\frac{389}{512},-\frac{263}{256}, \frac{53}{16},-\frac{1}{8},-\frac{5}{2}, 1\right)^{t}$, and the linear system

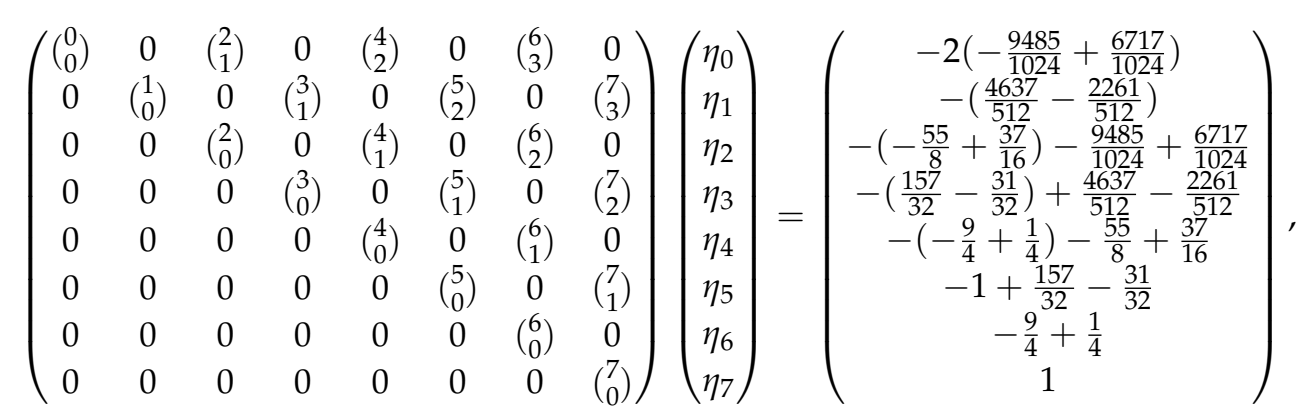

has solution $\left(\frac{9}{16}, \frac{15}{16},-\frac{377}{64}, \frac{1}{64}, \frac{151}{16},-\frac{65}{16},-2,1\right)^{t}$. Therefore, the polynomials $P_{6}(x)$ and $Q_{5}(x)$ of Theorem 3 are

$$
P_{6}(x)=x^{6}-\frac{5}{2}-\frac{x^{4}}{8}+\frac{53}{16} x^{3}-\frac{263}{256} x^{2}-\frac{389}{512} x+\frac{105}{512}
$$

and

$$
\left(x^{2}-4\right) Q_{5}(x)=x^{7}-2 x^{6}-\frac{65}{16} x^{5}+\frac{151}{16} x^{4}+\frac{x^{3}}{64}-\frac{377}{64} x^{2}+\frac{15}{16} x+\frac{9}{16} .
$$

It is not difficult to show that $P_{6}$ and $Q_{5}$ have real, simple and interlaced roots in $(-2,2)$ and therefore $S_{11}$ is a Schur polynomial. The zeros of the polynomials involved are plotted in the following Figure 1.

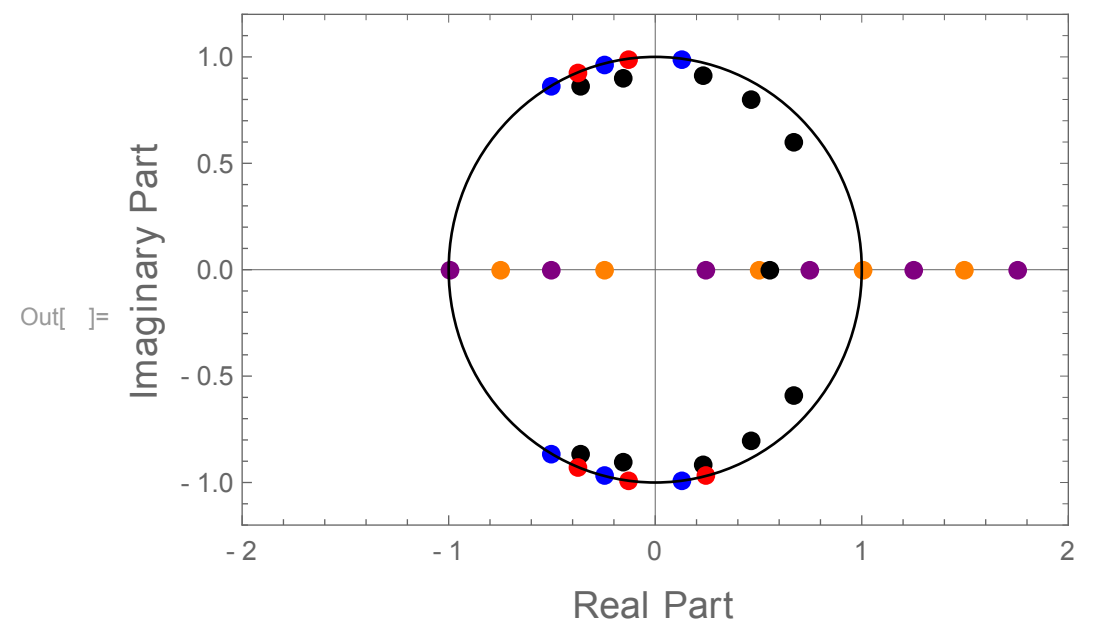

- Zeros of $\mathrm{P}_{6}(\mathrm{x})$

- Zeros of $Q_{5}(x)$

- Zeros of $P_{6}(z+1 / z)$

- Zeros of $Q_{5}(z+1 / z)$

- Zeros of $S_{11}(z)$

Figure 1. Zeros of $P_{6}(x), P_{6}(z+1 / z), Q_{5}(x), Q_{5}(z+1 / z)$ and $S_{11}(z)$.

On the other hand, if $T$ denotes the transformation defined by Equation (5), define

$$
\hat{S}(x):=T S(z)=(x-1)^{n} S\left(\frac{x+1}{x-1}\right) .
$$

Then we have the following straightforward Schur analogue of Markov's parameters criterion for Hurwitz polynomials.

Proposition 1. Let $S_{m}(z)$ be a polynomial of degree $m$ with real coefficients such that $S_{m}(1) \neq 0$. Then, $S_{m}(z)$ is a Schur polynomial if and only if the constants $s_{i}$ of the expansion

$$
\frac{\hat{S}_{m}^{a}\left(x^{1 / 2}\right)}{x^{1 / 2} \hat{S}_{m}^{s}\left(x^{1 / 2}\right)}= \begin{cases}\frac{s_{0}}{x}-\frac{s_{1}}{x^{2}}+\frac{s_{2}}{x^{3}}-\ldots+\frac{s_{2 n-2}}{x^{2 n-1}}-\frac{s_{2 n-1}}{x^{2 n}}+\ldots, & \text { if } \quad m=2 n \\ s_{-1}+\frac{s_{0}}{x}-\frac{s_{1}}{x^{2}}+\frac{s_{2}}{x^{3}}-\ldots+\frac{s_{22-2}}{x^{2 n-1}}-\frac{s_{2 n-1}}{x^{2 n}}+\ldots, & \text { if } \quad m=2 n+1 .\end{cases}
$$


are such that the matrices

$$
H_{n-1}=\left(\begin{array}{cccc}
s_{0} & s_{1} & \ldots & s_{n-1} \\
s_{1} & s_{2} & \ldots & s_{n} \\
\vdots & \vdots & \vdots & \vdots \\
s_{n-1} & s_{n} & \ldots & s_{2 n-2}
\end{array}\right), \quad H_{n-1}^{(1)}=\left(\begin{array}{cccc}
s_{1} & s_{2} & \ldots & s_{n} \\
s_{2} & s_{3} & \ldots & s_{n+1} \\
\vdots & \vdots & \vdots & \vdots \\
s_{n} & s_{n+1} & \ldots & s_{2 n-1}
\end{array}\right),
$$

are positive definite. If $m=2 n+1$ it is also required that $s_{-1}>0$.

Proof. $S_{m}(z)$ is a Schur polynomial if and only if

$$
\hat{S}_{m}^{s}\left(x^{1 / 2}\right)=h(x), \quad x^{-1 / 2} \hat{S}_{m}^{a}\left(x^{1 / 2}\right)=g(x),
$$

where $f(x)=h\left(x^{2}\right)+x g\left(x^{2}\right)$ is a Hurwitz polynomial (see [22]). The result follows from Markov's parameters criterion.

Notice that the above means that under the action of the Möbius transformation Equation (5), the symmetric (resp. asymmetric) part of a Schur polynomial is related to the even (resp. odd) part of a Hurwitz polynomial.

Example 3. Consider the polynomial $S_{4}(z)=z^{4}-2 z^{3}+\frac{67}{36} z^{2}-\frac{31}{36} z+\frac{13}{72}$. Then,

$$
S_{4}^{s}=\frac{85}{144} z^{4}-\frac{103}{72} z^{3}+\frac{67}{36} z^{2}-\frac{103}{72} z+\frac{85}{144}, \quad \text { and } \quad S_{4}^{a}=\frac{59}{144} z^{4}-\frac{41}{72} z^{3}+\frac{41}{72} z-\frac{59}{144},
$$

and by applying Equation (5) we get

$$
\hat{S}_{4}^{s}=\frac{1}{72}\left(425+242 z^{2}+13 z^{4}\right), \quad \hat{S}_{4}^{a}=\frac{50}{9} z+z^{3} .
$$

Thus,

$$
\frac{\hat{S}_{4}^{a}\left(x^{1 / 2}\right)}{x^{1 / 2} \hat{S}_{4}^{s}\left(x^{1 / 2}\right)}=\frac{400+72 x}{425+242 x+13 x^{2}}=\frac{72}{13 x}-\frac{12,224}{169 x^{2}}+\frac{2,560,408}{2197 x^{3}}-\frac{552,081,136}{28,561 x^{4}}+\cdots,
$$

and the matrices

$$
H_{1}=\left(\begin{array}{cc}
\frac{72}{13} & \frac{12,224}{169} \\
\frac{12,224}{169} & \frac{2,560,408}{2197}
\end{array}\right), \quad H_{1}^{(1)}=\left(\begin{array}{cc}
\frac{12,224}{169} & \frac{2,560,408}{2197} \\
\frac{2,560,408}{2197} & \frac{552,081,136}{28,561}
\end{array}\right)
$$

are positive definite. As a consequence, $S_{4}(z)$ is a Schur polynomial.

\section{Conclusions and Further Remarks}

We have obtained a criterion in Theorem 3 to determine whether or not a given polynomial $S_{m}(z)$ is Schur. To the best of our knowledge, this method is not known in the literature. Notice that it is a Hermite-Biehler type criterion in the sense that it involves verifying the interlacing of a pair of polynomials that are obtained from $S_{m}$. Our result is an improvement of Theorem 1, since there it is required to verify the interlacing of two polynomials of degree $n$, whereas in our method the interlacing is verified for polynomials of degree $m / 2($ resp. $(m+1) / 2)$ when $n$ is even (resp. odd). As an immediate consequence, we also obtain a simple necessary condition for Schur stability in Corollary 1. A criterion related to the positivity of Hankel matrices is obtained in Proposition 1.

Author Contributions: Formal analysis, L.E.G., N.M. and G.R.; Funding acquisition, G.R.; Software, N.M.; Writing-original draft, L.E.G. and N.M.; Writing—review and editing, L.E.G. and G.R. All authors have read and agreed to the published version of the manuscript. 
Funding: The work of the first author was supported by México's Conacyt Grant $287523 . \quad$ The work of Gerardo Romero and Noé Martínez was supported by Universidad Autónoma de Tamaulipas, under grants: P/PROFEXCE-2020-28MSU0010B-20, PROINNOVA-2018-250105, PROINNOVA-2018-250586, PROINNOVA-2018-250540, PROINNOVA-2018-250113, PROINNOVA-2018-250117, PROINNOVA-2018-250160 and PROINNOVA-2018-250255.

Conflicts of Interest: The authors declare no conflict of interest.

\section{References}

1. Åström, K.J.; Wittenmark, B. Computer-Controlled Systems; Prentice Hall: Upper Saddle River, NJ, USA, 1990.

2. Kuo, B.C. Digital Control Systems; Oxford University Press: Oxford, UK, 1992.

3. Jalili-Kharaajoo, M.; Araabi, B.N. The Schur stability via the Hurwitz stability analysis using a biquadratic transformation. Automatica 2005, 41, 173-176. [CrossRef]

4. Fleming, R.; Grossman, G.; Lenker, T.; Narayan, S. On Schur D-Stable matrices. Linear Algebra Appl. 1988, 279, 39-50. [CrossRef]

5. Xu, H.; Datta, A.; Bhattacharyya, S.P. Computation of all stabilizing PID gains for digital control systems. IEEE Trans. Autom. Control 2001, 46, 647-652. [CrossRef]

6. Jury, E.I.; Blanchard, J. A stability test for linear discrete system in table form. Proc. IRE 1961, 49, 1947-1948.

7. Jury, E.I.; Blanchard, J. A stability test for linear discrete system using a simple division. Proc. IRE 1961, 49, 1948-1949.

8. Choo, Y. An elementary proof of the Jury test for real polynomials. Automatica 2011, 47, 249-252. [CrossRef]

9. Pérez, F.; Abdallah, C.; Docampo, D. Extreme-point stability test for discrete-time polynomials. In Proceedings of the 31st Conference on Decision and Control, Tucson, AZ, USA, 16-18 December 1992; pp. 1552-1553.

10. Shiomi, K.; Otsuka, N.; Inaba, H.; Ishii, R. The property of bilinear transformation matrix and Schur stability for a linear combination of polynomials. J. Frankl. Inst. 1999, 336, 533-541. [CrossRef]

11. Ackermann, J.E.; Barmish, B.R. Robust Schur stability of a polytope of polynomials. IEEE Trans. Autom. Control 1988, 33, 984-986. [CrossRef]

12. Bose, N.K.; Jury, E.I.; Zeheb, E. On robust hurwitz and Schur polynomials. IEEE Trans. Autom. Control 1988, 33, 1166-1168. [CrossRef]

13. Greiner, R. Necessary conditions for Schur-stability of interval polynomials. IEEE Trans. Autom. Control 2004, 49, 740-744. [CrossRef]

14. Kraus, F.; Mansour, M.; Jury, E.I. Robust Schur stability of interval polynomials. IEEE Trans. Autom. Control 1992, 37, 141-143. [CrossRef]

15. Shih, M.H.; Pang, C.T. Simultaneous Schur stability of interval matrices. Automatica 2008, 44, $2621-2627$. [CrossRef]

16. Pastravanu, O.; Matcovschi, M.H. Sufficient conditions for Schur and Hurwitz diagonal stability of complex interval matrices. Linear Algebra Appl. 2015, 467, 149-173. [CrossRef]

17. Torres-Muñoz, J.A.; Rodríguez-Ángeles, E.; Kharitonov, V.L. On Schur stable multivariate polynomials. IEEE Trans. Circuits Syst. I Regul. Pap. 2006, 53, 1166-1173. [CrossRef]

18. Dumitrescu, B.; Chang, B.C. Robust Schur stability with polynomial parameters. IEEE Trans. Circuits Syst. II Exp. Briefs 2006, 53, 535-537. [CrossRef]

19. Dabbene, F.; Henrion, D.; Lagoa, C.M. Simple approximations of semialgebraic sets and their applications to control. Automatica 2017, 78, 110-118. [CrossRef]

20. Gantmacher, F.R. The Theory of Matrices; Chelsea Publishing Co.: New York, NY, USA, 1959; Volumes 1-2.

21. Lancaster, P.; Tismenetsky, M. The Theory of Matrices and Applications; Academic Press: Cambridge, MA, USA, 1985.

22. Bhattacharyya, S.P.; Chapellat, H.; Keel, L.H. Robust Control: The Parametric Approach; Prentice-Hall: Upper Saddle River, NJ, USA, 1995.

23. Hermite, C. Sur le nombre des racines dune equation algebrique comprise entre des limites donnes. J. Reine Angew. Math. 1856, 52, 39-51.

24. Bäckström, T.; Magi, C. Properties of line spectrum pair polynomials-A review. Signal Process. 2006, 86, 3286-3298. [CrossRef] 
25. Szegő, G. Orthogonal Polynomials, 4th ed.; American Mathematical Society Colloquium Publications: Providence, RI, USA, 1975; Volume 23.

26. Chihara, T.S. An Introduction to Orthogonal Polynomials; Mathematics and Its Applications Series; Gordon and Breach: New York, NY, USA, 1978.

27. Simon, B. Orthogonal Polynomials on the Unit Circle; American Mathematical Society Colloquium Publications: Providence, RI, USA, 2005; Volume 54.

28. Ismail, M.E.H. Classical and Quantum Orthogonal Polynomials in One Variable; Encyclopedia of Mathematics and Its Applications; Cambridge University Press: Cambridge, UK, 2005; Volume 98.

29. Huertas, E.J.; Marcellán, F.; Rafaeli, F.R. Zeros of orthogonal polynomials generated by canonical perturbations of measures. Appl. Math. Comput. 2012, 218, 7109-7127. [CrossRef]

30. Brezinski, C. Padé-Type Approximation and General Orthogonal Polynomials; International Series of Numerical Mathematics, SO; Birkhäuser Verlag: Basel, Switzerland, 1980.

31. Genin, I.V. Euclid algorithm, orthogonal polynomials, and generalized routh-hurwitz algorithm. Linear Algebra Appl. 1996, 246, 131-158. [CrossRef]

32. Holtz, O. Hermite-Biehler, Routh-Hurwitz, and total positivity. Linear Algebra Appl. 2003, 372, $105-110$. [CrossRef]

33. Lange, L.J. Continued fraction applications to zero location. In Analytic Theory of Continued Fractions II, Lecture Notes in Math., Vol. 1199, Proceedings of a Seminar-Worship, Pitlochry and Aviemore, Scotland, UK, 13-29 June 1985; Thron, W.J., Ed.; Springer: Berlin/Heidelberg, Germany, 1986; pp. 220-262.

34. Martínez, N.; Garza, L.E.; Aguirre-Hernández, B. On sequences of Hurwitz polynomials related to orthogonal polynomials. Linear Multilinear A 2019, 67, 2191-2208. [CrossRef]

35. Arceo, A.; Garza, L.E.; Romero, G. Robust stability of hurwitz polynomials associated with modified classical weights. Mathematics 2019, 7, 818. [CrossRef]

36. Simon, B. Szegö's Theorem and Its Descendants: Spectral Theory for L2 Perturbations of Orthogonal Polynomials; M. B. Porter Lectures; Princeton University Press: Princeton, NJ, USA, 2011.

(c) 2020 by the authors. Licensee MDPI, Basel, Switzerland. This article is an open access article distributed under the terms and conditions of the Creative Commons Attribution (CC BY) license (http:/ / creativecommons.org/licenses/by/4.0/). 